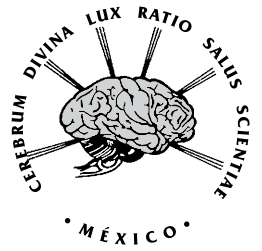

\title{
Reseña de Consultoría en Enfermería Neurológica
}

\section{Review Neurological Nursing Consultancy}

\author{
MCE. Angélica Guadalupe Dávalos-Alcázar*
}

* Editora de la Revista de Enfermería Neurológica

En México, la consultoría de enfermería, como es conocida al día de hoy, se realiza por profesionales de la disciplina, dando inicio en 1997. En ese momento se llevó a efecto, principalmente, en el primer nivel de atención y uno de los lugares específicos fue el Centro de Investigación Materno Infantil del Grupo de Estudios al Nacimiento (CIMI-Gen) de la Ciudad de México; así mismo, se estructuraron diferentes Programas Integrados de Salud como fue (PREVENIMSS) del Instituto Mexicano del Seguro Social (IMSS), donde se inició el desarrollo de acciones de carácter preventivo y de rehabilitación cuyo objetivo fundamental es mejorar la salud mediante la atención en la consulta externa; sin embargo, estas acciones fueron desarrollándose posteriormente en el Segundo Nivel de Atención, siendo al día de hoy aplicadas en el Hospital General de México.

A partir de 2011, se emplea el Modelo de Consultoría en Enfermería Neurológica (MCEN) en el Instituto Nacional de Neurología y Neurocirugía (INNN), mismo que fue facultado e implementado el día 13 de septiembre de 2011 con el fin de beneficiar al enfermo por medio de la relación enfermera-paciente-familia. Este modelo es aplicado por profesionales enfermeros especializados en el cuidado neurológico, neuroquirúrgico y neuropsiquiátrico, los cuales cuentan con experiencia clínica y pensamiento crítico, así como conocimientos y habilidades para proporcionar consulta de alta especialidad. Los requisitos para incorporarse al programa son contar con posgrado académico de especialista y una experiencia clínica neurológica de al menos cinco años. Entre las funciones que realiza el especialista en la consulta está, en primer término, la valoración de la persona; con ello se establecen, a partir de un pensamiento crítico, los diagnósticos de enfermería reales y potenciales para planear de manera individualizada las características y directrices del cuidado que tendrá cada persona; tales directrices deberán ser ejecutadas cuidadosamente para dar así seguimiento y evaluar la instrucción y capacitación al familiar, dependiendo del tipo de padecimientos neurológicos, neuroquirúrgicos y psiquiátricos presentes y las limitantes propias de cada paciente. De esta forma se contribuye en la mejora de calidad en la atención para cubrir la alta demanda de servicios de salud, disminuyendo con ello los reingresos hospitalarios y por tanto los costos, incentivando la adherencia terapéutica y previniendo los posibles riesgos para la salud. Esto mejora los estilos y calidad de vida y ayuda en la rehabilitación; todo ello a partir del proceso enfermero.

En la práctica de enfermería especializada se han logrado en los últimos tres años avances significativos; uno de ellos es el modelo de consultoría, lo que demanda en el profesional enfermero una alta competencia en materia de neurociencia, abarcando aspectos en donde la calidad en el cuidado está de manifiesto.

Nuestra institución se ha destacado por ser un centro de enseñanza de distintos profesionistas en el Área de las Ciencias Neurológicas y muy en especial de aquellos que proporcionan atención directa a los usuarios. Uno de los actores principales en esto es el personal de Enfermería, que participa en los servicios de hospitalización y continúa su quehacer mediante asesoramiento profesional, entrevistándose con el enfermo y orientándolo en cuanto a la prevención y la promoción de la salud, así como en la realización de cuidados especializados para su atención y rehabilitación. De esta forma, tendrá mayor seguridad y calidad de vida cuando ya se encuentre en su domicilio. 
Enf Neurol (Mex) • 2014 • Vol. 13 • Núm. 3 • 105-106

Los resultados hasta ahora obtenidos en la Consultoría en Enfermería Neurológica han sido satisfactorios. Los pacientes atendidos en el periodo de septiembre de 2011 a diciembre de 2013 son 127; los principales motivos por los que asistieron a la Consulta de Enfermería Neurológica fueron el mal apego y la orientación sobre cuidados especiales en el hogar. En la evaluación que se les realizó, se les preguntó si acudirían nuevamente a la consultoría y el $87.5 \%$ respondió que sí.

El impacto en los pacientes que han acudido a la consulta ha sido satisfactorio, ya que se está actuando desde diferentes ángulos para ayudar a resolver sus necesidades humanas y por tanto tener una mejor calidad de vida; esto también ayuda al instituto, pues disminuye el número de reingresos por mal apego al tratamiento y complicaciones por falta de cuidado en el hogar.

Hoy contamos con un grupo de profesionales que dan cauce y consolidación a la consultoría institucional.

Grupo de consultoras:

Rocio Valdez Labastida

Patricia Zamora Ruiz

Ma. Verónica Balcázar Martínez

Silvia Alejandro Escobar

Ma. Guadalupe Nava Galán

Estela Reyes Chávez

Teresa Martínez Acosta

Mirna Desales Ramos

Magdalena Castillo Pérez

Laura Oralia Torres López

Karla Cansino Morales

Marco Antonio Ortega Ventura

El profesionista enfermero incursiona día con día en nuevos rubros que posicionan y proyectan su quehacer cotidiano desde la perspectiva de la alta especialización en el cuidado, por lo que asume y está facultado profesional y directivamente para desplegar de la consultoría en enfermería neurológica.
Agradecimiento por la información proporcionada:

Rocio Valdez Labastida

Patricia Zamora Ruiz

Ma. Verónica Balcázar Martínez

Silvia Alejandro Escobar

Ma. Guadalupe Nava Galán

\section{BIBLIOGRAFÍA}

1. Ley General de Salud.

2. Organización Mundial de la Salud: Programa de acción sobre medicamentos esenciales. Ginebra: Organización Mundial de la Salud; 1998.

3. Sánchez B. Identidad y empoderamiento de la profesión de enfermería. Avances en Enfermería. 2002; 20 (1): 22-32.

4. Vargas-García C. Centro de Investigación Materno Infantil del Grupo de Estudios al Nacimiento México (CIMI-Gen), Consultado el 30 de agosto de 2011, Disponible en: http:// www.comminit.com/la/node/37725.

5. Organización Panamericana de la Salud.

6. Ballesteros MM, Jiménez L. La enfermería, una profesión de servicio a las personas. Metas de Enfermería. 2003; 6 (59): 65.

7. CIE. La definición de enfermería. Consultado en marzo de 2011. Disponible en: http://www.actualidad.e nfermundi.com/ enfi n/enfi ncie/index.asp.

8. Castro-Serralde E, Padilla-Zárate MP, Solís-Flores L. Consejería personalizada en enfermería en el Hospital General de México. Rev Med Hosp Gen Mex. 2009; 72 (4): 228-230.

9. Restrepo de ACM. Simbolismo de la profesión. En: Historia de la Facultad de Enfermería de la Universidad de Antioquia. Medellín. 1997; 162-198.

10. Alberdi RM. Las enfermeras para el tercer milenio. Rol de Enfermería. 1993; 178: 43-50.

11. Castrillón MC. La disciplina de Enfermería. En: Desarrollos y perspectivas de la profesión de Enfermería. Inv Edu Enfer. 2000; 10 (2): 53-56.

12. Muñoz-Hernández $O$. Programas Integrados de Salud. Rev Med Inst Mex Seguro Soc. 2006; 44 (Supl 1): S1-S2.

13. Marriner T. Teorías y modelos. 6a. ed. España: Elsevier; 2007: 3-14.

Dirección para Correspondencia:

MCE. Angélica Guadalupe Dávalos-Alcázar

E-mail: angelicadavalos10@gmail.com 